EPJ Web of Conferences 19, 05004 (2012)

DOI: $10.1051 /$ epjconf/20121905004

(C) Owned by the authors, published by EDP Sciences, 2012

\title{
Solar neighbourhood and Galactic disc(s): New constraints
}

\author{
L. Casagrande ${ }^{a}$ and R. Schönrich \\ Max-Planck-Institute for Astrophysics, Garching, Germany
}

\begin{abstract}
We present a re-analysis of the Geneva-Copenhagen survey, based on improved effective temperature and metallicity scales, which also provide a better match to theoretical isochrones. The latter are used for a Bayesian investigation on stellar ages. With respect to previous analyses, our stars are on average $100 \mathrm{~K}$ hotter and 0.1 dex more metal rich, which shifts the peak of the metallicity distribution function around the solar value. From Strömgren photometry we are able to derive for the first time a proxy for alpha elements, which enables us to perform a tentative dissection of the chemical thin and thick disc. We find evidence for the latter being composed of an old, mildly but systematically alpha-enhanced population that extends to super solar metallicities, in agreement with spectroscopic studies. Our analysis suggests a strong interplay among ages, abundances and kinematics of stars.
\end{abstract}

\section{INTRODUCTION}

Late-type dwarf stars are long-lived objects and can be regarded as snapshots of the stellar populations that are formed at different times and places over the history of our Galaxy. Not only their kinematics carry residual information on their dynamical histories, but their atmospheres retain a fossil record of the composition of elements in the interstellar medium at the time and place of their formation. Therefore, F, G, and - to a lesser extent - K dwarfs have been historically used to study various aspects of the chemical evolution of the Milky Way, and the solar neighbourhood in particular e.g. [1-3]. While spectroscopic studies allow detailed abundance investigations, they are still limited to small samples of a few hundred or about a thousand stars at most, and must use sophisticated kinematic selections to sample significant numbers of members belonging to different Galactic subpopulations e.g. [4]. On the contrary, photometric catalogues usually have access to several thousands of stars - such as the GenevaCopenhagen Survey [3] - and thus are largely free from selection biases, but have to pay for this by being only able to derive one single parameter for metallicity (and no detailed elemental abundances) from photometry.

Here, we present a revision of the astrophysical parameters in the Geneva-Copenhagen Survey (GCS) with the new effective temperature $\left(T_{\text {eff }}\right)$ scale presented in [5] as a starting point to derive new metallicities and ages. We improve not only on the accuracy, i.e. reduce zeropoint systematics, but also the precision by reducing internal errors stemming from photometric transformations. These improvements turn out to be crucial to provide more stringent observational constraints on Galactic chemical evolution theories and hence on the history of the Milky Way. In fact, a knowledge of the metallicity distribution together with Galactic abundance gradients can improve our understanding of the impact and shape of the stellar migration process in the Galactic disc [6, 7]. Because models including radial migration relax the classical tight correlation between age and metallicity, this relation becomes effectively an additional constraint independent from the metallicity distribution. In addition,

\footnotetext{
a e-mail: luca@mpa-garching.mpg.de
}

This is an Open Access article distributed under the terms of the Creative Commons Attribution-Noncommercial License 3.0, which permits unrestricted use, distribution, and reproduction in any noncommercial medium, provided the original work is properly cited. 


\section{EPJ Web of Conferences}

being able for the first time to derive an estimate for alpha elements from Strömgren photometry allows for a tentative dissection of the chemical thin and thick disc with a sample which exceeds the largest spectroscopic studies available so far by more than an order of magnitude.

\section{DETERMINATION OF ASTROPHYSICAL PARAMETERS}

The Geneva-Copenhagen Survey provides the ideal database for studies dealing with Galactic chemical and dynamical evolution: it is kinematically unbiased, all its stars have highly homogeneous Strömgren photometry, from which stellar abundance information can be readily derived and merging this catalogue with Tycho 2 and 2 MASS provides the multi-band optical and infrared photometry needed to derive $T_{\text {eff }}$ via the infrared flux method.

\subsection{Effective temperatures}

The infrared flux method described in [5, 8] not only improves the accuracy of the zeropoint of the derived stellar parameters, but also their precision by employing Tycho2 $B_{T} V_{T}$ and 2 MASS $J H K_{S}$ photometry to simultaneously recover the bolometric flux and the effective temperature of each star. Thus, it is well suited to be applied to the Geneva-Copenhagen catalogue directly, avoiding the use of colour calibrations as well as transformations among different photometric systems. Compared to previous GCS calibrations, our effective temperatures are on average hotter by about 80-100 K; at the same time the improved methodology often reduces the intrinsic scatter uncertainty per star below $100 \mathrm{~K}$. As we see later, the HR diagram constructed using our newly derived $T_{\text {eff }}$ scale matches very well that predicted by stellar models for evolved $\mathrm{F}$ and $\mathrm{G}$ dwarfs $[9,10]$, and the possible uncertainties which might still affect the cool end of the main sequence $[11,12]$ are thus of no concern for the sake of the GCS analysis.

\subsection{Metallicities}

Since the adopted effective temperature scale has immediate consequences on abundances, we recalibrated Strömgren indices versus stellar metallicities using a sample of nearly 1500 stars with highresolution spectroscopic abundances derived adopting $T_{\text {eff }}$ consistent with ours. For the first time we are able to derive rough estimates for alpha elements $(\alpha \mathrm{Fe})$ from Strömgren photometry. The method becomes less reliable for increasingly hotter objects and also for metal-poor stars (roughly below -1 dex), but focusing on disc stars gives a reasonable guidance on the relative alpha enhancements for the whole sample. The new metallicity scale was then checked against open clusters and a moving group, showing indeed a high degree of internal consistency with a suggested intrinsic scatter below 0.10 dex in $[\mathrm{Fe} / \mathrm{H}]$. The recently measured $u v b y$ solar colours [13] finally corroborate the agreement between the temperature and metallicity scales.

\subsection{Ages}

Revising metallicities and effective temperatures also affects age and mass estimates for the stars. Compared to previous studies, our improved effective temperatures are hotter and the large systematic discrepancies between theoretical isochrones and observed data that plagued e.g., [3] almost entirely disappear, without having to introduce metallicity-dependent temperature shifts to reconcile isochrones with data. This greatly reduces the risk of introducing an artificial age-metallicity relationship.

Naïve fits to isochrones lead to severe biases, e.g. the terminal age bias. This happens because some places on isochrones are more densely populated than others because of the mapping from mass to colours/luminosity owing to the initial mass function and to the time scales involved in stellar evolution. Just looking for the closest match ignores these facts and might erroneously place too many stars into 

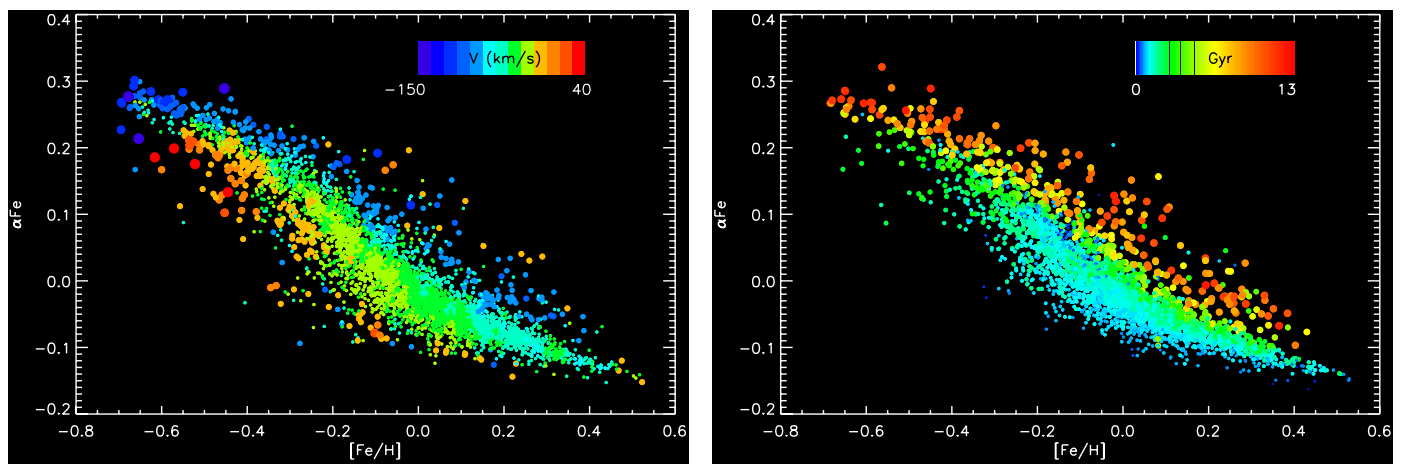

Figure 1. Left: photometric alpha estimates $\alpha \mathrm{Fe}$ versus $[\mathrm{Fe} / \mathrm{H}]$ according to the rotational velocities of the stars (represented by different colours). Right: same as in left panel, with different colours now representing stars of different ages.

sparsely populated regions of the HR diagram. Biases of this kind can be accounted for by taking a Bayesian approach as described in [14] and [15]. Here we implement the same technique, where isochrones provide a grid for calculating the probability distribution function of the parameters of any given star. In order to study differences between different isochrones, we used grids of the BASTI [16] and Padova [17] isochrones, obtaining similar results.

\section{CLASSICAL OBSERVATIONAL CONSTRAINTS}

Given its nearly volume complete nature, the GCS is well suited for the study of the metallicity distribution function (MDF) in the solar neighbourhood. As a consequence of our revised metallicity scale, the peak of the MDF is only slightly subsolar, thus making the Sun a completely average star given its metallicity (see also [18]).

The MDF has been historically used to constrain the gas infall rate [19], but slicing it into different age intervals suggests that old stars are also a relevant ingredient in describing the wings of the MDF. A natural explanation to this is provided by radial migration, where the solar neighbourhood is not only assembled from local stars, following a local age-metallicity relation, but also originating from the inner (more metal-rich) and outer (more metal-poor) Galactic disc that have migrated to the present position on different timescales [6, 20].

The GCS has a small spatial extent, but in principle its large number of stars would still allow for an estimate of the radial metallicity gradients in the Galactic disc. This can mostly be achieved by using the azimuthal velocities of stars which give the abundance gradient in angular momentum, which is by the flat rotation curve of the Milky Way disc directly related to the Galactocentric radius they stem from. However, its exact value depends on the adopted kinematic or metallicity cuts to avoid halo contamination, as well as the age interval considered. Difficulties in estimating e.g. the interdependence between age and kinematic cuts (as stars with increasing asymmetric drift are preferentially older, see also Fig. 1) as well as the increasing scatter in the age-metallicity and in the age-dispersion relation [21] further complicate the picture. Thus, despite being extremely powerful in constraining chemical models for the disc, metallicity gradients should always be taken cum grano salis.

\section{NEW OBSERVATIONAL CONSTRAINTS}

The Milky Way is usually dissected into two-component disc, which was first proposed to fit the vertical density profile derived from star counts [22, 23]. Disentangling the nature and origin of these 
components is therefore highly relevant for understanding galaxy formation. Though limited to the solar neighbourhood, the GCS can provide important insights into this puzzle, because it is essentially free from kinematic selections. Our metallicities and alpha estimates provide for the first time a way to investigate this with a more complete sample.

Fig. 1 shows the $\alpha \mathrm{Fe}$ vs. $[\mathrm{Fe} / \mathrm{H}]$ using our revised GCS sample, where stars with different rotation velocities $(V)$ and ages are depicted by different colours. Clearly, this is only a rough criterion for the division and this selection is not stringent in targeting single disc "components", yet a striking difference appears. At each metallicity, the stars with highly negative $V$ velocities, or at high/large asymmetric drift (bluemost colours) have higher average $\alpha \mathrm{Fe}$. This can be expected because stars with such a large asymmetric drift should be significantly older than the remaining population (because of the asymmetric drift-dispersion and the age-dispersion relations), which is confirmed by their ages in the right panel, which are indeed older (redder colours). Our analysis thus clearly confirms a similar result drawn by [24] from a smaller spectroscopic sample.

Because of the tight age-metallicity relation in chemical evolution models without radial migration, older and more alpha-enhanced stars are expected to be more metal-poor. Yet Fig. 1 rather tells the opposite, at a given alpha value the the old and alpha-rich stars are on average more metal-rich than the population with positive rotation velocities. The emergence of a metal-rich, old thick disc was already noticed in spectroscopic sample of [25]. This apparently surprising behaviour is however readily explained if there is no strong age-metallicity dependence, as is the case in radial migration models, and if the lagging metal-rich population comprises - to some extent at least - objects from the inner disc, which are more metal-rich thanks to the Galactic metallicity gradient [6].

\section{References}

[1] B.A. Twarog, ApJ, 242, 242 (1980)

[2] B. Edvardsson, J. Andersen, B. Gustafsson, D.L. Lambert, P.E. Nissen, J. Tomkin, A\&A, 275, 101 (1993)

[3] B. Nordström, M. Mayor, J. Andersen, J. Holmberg, F. Pont, B.R. Jørgensen, E.H. Olsen, S. Udry, N. Mowlavi, A\&A, 418, 989 (2004)

[4] T. Bensby, A.R. Zenn, M.S. Oey, S. Feltzing, ApJL, 663, L13 (2007)

[5] L. Casagrande, I. Ramírez, J. Meléndez, M. Bessell, M. Asplund, A\&A, 512, A54+ (2010)

[6] R. Schönrich, J. Binney, MNRAS, 396, 203 (2009)

[7] R. Schönrich, J. Binney, MNRAS, 399, 1145 (2009)

[8] L. Casagrande, L. Portinari, C. Flynn, MNRAS, 373, 13 (2006)

[9] D.A. VandenBerg, L. Casagrande, P.B. Stetson, AJ, 140, 1020 (2010)

[10] C.M. Brasseur, P.B. Stetson, D.A. VandenBerg, L. Casagrande, G. Bono, M. Dall'Ora, AJ, 140, $1672(2010)$

[11] L. Casagrande, C. Flynn, L. Portinari, L. Girardi, R. Jimenez, MNRAS, 382, 1516 (2007)

[12] L. Portinari, L. Casagrande, C. Flynn, MNRAS, 406, 1570 (2010)

[13] J. Meléndez, W.J. Schuster, J.S. Silva, I. Ramírez, L. Casagrande, P. Coelho, A\&A, 522, A98+ (2010)

[14] F. Pont, L. Eyer, ApJ, 351, 487 (2004)

[15] B.R. Jørgensen, L. Lindegren, A\&A, 436, 127 (2005)

[16] A. Pietrinferni, S. Cassisi, M. Salaris, S. Percival, J.W. Ferguson, ApJ, 697, 275 (2009)

[17] G. Bertelli, L. Girardi, P. Marigo, E. Nasi, A\&A, 484, 815 (2008)

[18] M. Asplund, N. Grevesse, A.J. Sauval, P. Scott, ARA\&A, 47, 481 (2009)

[19] D. Lynden-Bell, Vistas in Astronomy 19, 299 (1975)

[20] R. Roškar, V.P. Debattista, T.R. Quinn, G.S. Stinson, J. Wadsley, ApJL, 684, L79 (2008) 
Assembling the Puzzle of the Milky Way

[21] L. Casagrande, R. Schönrich, M. Asplund, S. Cassisi, I. Ramírez, J. Meléndez, T. Bensby, S. Feltzing, A\&A, 530, A138+ (2011)

[22] Y. Yoshii, PASJ, 34, 365 (1982)

[23] G. Gilmore, N. Reid, MNRAS, 202, 1025 (1983)

[24] M. Haywood, MNRAS, 388, 1175 (2008)

[25] S. Feltzing, T. Bensby, Physica Scripta Volume T 133(1), 014031 (2008) 